\title{
The Chemical Constitution of Thyroxine.
}

A LTHOUGH the stimulant action of the thyroid gland upon the general metabolism of the body has been recognised for more than a generation, yet it is only within the last decade that the isolation of a pure crystalline principle, possessing the stimulating effects of the whole gland, has been successful. This was first accomplished by Kendall in I9I4; since then this author has described a number of the derivatives of the pure substance, which he called "thyroxin," and has suggested a structural formula for it. Our knowledge of it has now been carried a step further by Harington, who has improved the method of extraction so that it gives a much higher yield than Kendall's method, and has proceeded, with the larger amount of material thus available, to determine its chemical constitution by methods of degradation and synthesis. Although at present synthesis has only been carried to the penultimate stage, there is every reason to hope, considering the way in which the author has unravelled the constitution of this compound, that the complete synthesis will soon be successful.

Before describing Harington's work, a few words may be said about the physiological properties of thyroxine. This subject, together with some recent work on its derivatives, has been reviewed by Kendall in his Chandler lecture ("Influence of the Thyroid Gland on Oxidation in the Animal Organism." By E. C. Kendall. New York: Columbia University Press; London: Oxford University Press, I925. Price $3 s$. net). The activity of thyroxine in stimulating metabolism is remarkable: $\mathbf{I} \mathrm{mgm}$. injected intravenously in a patient suffering with myxodema will increase the basal metabolic rate 2.5 per cent., corresponding to an increased output of $400 \mathrm{gm}$. carbon dioxide; the effect is, within limits, proportional to the dose. Moreover, the time relations of the response are characteristic. Thus, in a normal adult there is a latent period of six to eight hours; thereafter the metabolic rate rapidly increases but the maximum is not reached until the eighth or tenth day. The metabolism then returns slowly to normal, the rate of fall depending on the amount present in the body. A dose of 5-10 mgm. affects the metabolism for five or six weeks. It can replace the administration of dried thyroid gland in the treatment of cretinism and myxœdema; in a normal person, not only is the metabolism increased, but also the signs and symptoms of over-activity of the thyroid gland are also produced. Under normal conditions the thyroxine formed in the body appears to be responsible for about 40 per cent. of the metabolism, since complete thyroid deficiency causes a drop in the basal metabolic rate of this order.

The principle of the method of extraction from the thyroid gland is hydrolysis with alkali, followed by precipitation of the filtrate with acid, but whereas Kendall used 5 per cent. sodium hydroxide, Harington found that to per cent. barium hydroxide resulted in a greatly increased final yield (C. R. Harington, Biochem. Journ., vol. 20, 293, 1926). After about six hours' boiling of the dried thyroid in ten volumes of alkali, 60-70 per cent. of the total iodine appears in the filtrate; 40 per cent. of this can be precipitated on acidification as a creamy flocculent precipitate. The latter requires further hydrolysis with the alkali, when about half the iodine remains in solution and half is carried down with the insoluble barium salts ; the latter contain the thyroxine. The precipitate is boiled in alkaline sodium sulphate solution to remove the barium and the filtrate precipitated with acid. The precipitate of crude thyroxine can be further purified by solution in alcohol made alkaline with caustic soda and re-precipitating with acetic acid. Further material is obtained from the gland residues by boiling with caustic soda, adding sodium sulphate, filtering and precipitating the thyroxine in the filtrate with acid. After re-crystallisation, by dissolving in alkaline alcohol and adding acid, further purification is effected by dissolving in dilute hot sodium carbonate, when the sodium salt separates out on cooling. This is re-dissolved in alkaline alcohol and the thyroxine precipitated with acid. The pure substance crystallises in rosettes and sheaves of fine needles. On heating, it darkens at $220^{\circ} \mathrm{C}$. and melts with decomposition and evolution of iodine at $231^{\circ}-233^{\circ} \mathrm{C}$. It is insoluble in water and organic solvents; it is soluble in cold dilute solutions of alkali hydroxides, but insoluble in acids. It is soluble in 90 per cent. alcohol with the aid of either alkali hydroxide or mineral acid. The yield was about 0.027 per cent. of the fresh, or 0.12 per cent. of the dried gland, corresponding to about I 4 per cent. of the total iodine originally present; Kendall's yield was 0.00 I I per cent. of the fresh gland. The physiological activity of the material extracted by Harington's method was shown to be the same as that of thyroxine prepared by Kendall's process.

The compound contains 65.3 per cent. iodine; analysis led to the empirical formula $\mathrm{C}_{15} \mathrm{H}_{11} \mathrm{O}_{4} \mathrm{NI}_{4}$; Kendall's formula is $\mathrm{C}_{11} \mathrm{H}_{10} \mathrm{O}_{3} \mathrm{NI}_{3}$, and the difference between the two is due to the fact that Harington found a lower nitrogen content than Kendall. The former observer confirmed his analysis, however, on a commercial sample of thryoxine prepared according to Kendall's process. Kendall considers thyroxine to be triiodo-dihydro-oxindole propionic acid; Harington considers it to be the tetraiodo substitution derivative of the parahydroxy-phenyl ether of tyrosine.

The arguments in favour of the latter appear conclusive: the froducts of disintegration of the molecule have been identified; the foundation of the molecule has been synthesised and only requires the addition of the iodine atoms to become identical with the original thyroxine. The first step in the breakdown of the molecule is the removal of the iodine; this Harington accomplished by shaking a dilute alkaline solution in an atmosphere of hydrogen with a palladium hydroxide-calcium carbonate catalyst. The iodine was split off as potassium iodide, and the hydrogen taken up was exactly equivalent to the iodine removed. The resulting compound was called desiodo-thyroxine (Biochem. Journ., vol. 20, 300, I926). Its formula was found to be $\mathrm{HO}, \mathrm{C}_{6} \mathrm{H}_{4} \cdot \mathrm{O} \cdot \mathrm{C}_{6} \mathrm{H}_{4} \cdot \mathrm{CH}_{2} \cdot \mathrm{CHNH}_{2}$. COOH.

The interest of the subject appears to justify a somewhat detailed account of the successive steps in the disintegration and synthesis of desiodothyroxine. The facts that the latter gave positive Millon's and ninhydrin reactions, and that all the nitrogen was present as amino nitrogen, suggested that the compound was an a-amino-acid with at least one phenolic group; the proportions of carbon and hydrogen suggested the presence of two benzene rings. Fusion with potassium hydroxide produced parahydroxybenzoic acid, quinol in varying amounts according to the conditions, ammonia, oxalic acid, and a substance with the formula $\mathrm{C}_{13} \mathrm{H}_{12} \mathrm{O}_{2}$. The latter possessed only one phenolic group; the experiment thus suggested the presence of two benzene rings, one of which has a phenolic group in the para position to a side chain, from which a two-carbon fragment is split off as oxalic acid.

Exhaustive methylation of desiodo-thyroxin gave

NO. 2958 , VOL. I I 8 ] 
a product which lost trimethylamine on boiling with alkali and yielded an unsaturated acid, $\mathrm{C}_{16} \mathrm{H}_{14} \mathrm{O}_{4}$, containing only one methoxyl group. On oxidation with potassium permanganate, oxalic acid and $\mathrm{C}_{14} \mathrm{H}_{12} \mathrm{O}_{3}$ were formed; the latter was found to be an aldehyde which on further oxidation yielded an acid $\mathrm{C}_{14} \mathrm{H}_{12} \mathrm{O}_{4}$. Thus methylation shows that desiodothyroxin is almost certainly an amino acid, and that it contains only one phenolic group; the splitting off of oxalic acid suggests the presence of a three-carbon side chain. Now fusion with potash led to the conclusion that two benzene rings are present; if linked through a carbon atom, a ketone should have been formed instead of an aldehyde on oxidation of the methylated product; hence they must be linked either directly or through the oxygen atom, which is still unaccounted for. Considering the latter supposition the more probable, the author proceeded to meet the degradation by synthesis.

The steps in the disintegration by exhaustive methylation and in the subsequent synthesis may be rendered clearer if the following structural formulæ are given :

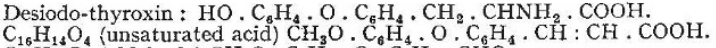
$\mathrm{C}_{14} \mathrm{H}_{12} \mathrm{O}_{3}$ (aldehyde) $\mathrm{CH}_{2} \mathrm{O} . \mathrm{C}_{6} \mathrm{H}_{4} \cdot \mathrm{O}_{6} \cdot \mathrm{C}_{6} \mathrm{H}_{4} . \mathrm{CHO}_{6}$.

$\mathrm{C}_{14} \mathrm{H}_{72} \mathrm{O}$, (saturated $\mathrm{CH}_{3} \mathrm{O} \cdot \mathrm{C}_{6} \mathrm{H}_{4} \cdot \mathrm{O} \cdot \mathrm{C}_{6} \mathrm{H}_{4} \cdot \mathrm{COOH}$

$\mathrm{C}_{13} \mathrm{H}_{12} \mathrm{O}_{2}$ (from potash fusion) $\mathrm{HO} \cdot \mathrm{C}_{6} \mathrm{H}_{4} \cdot \mathrm{O}_{6} \cdot \mathrm{C}_{6} \mathrm{H}_{4} \cdot \mathrm{CH}_{3}$.

The starting-point in the synthesis was parabromanisol, $\mathrm{CH}_{3} \mathrm{O} \cdot \mathrm{C}_{6} \mathrm{H}_{4} \cdot \mathrm{Br}$; on condensation with the potassium salt of paracresol in the presence of copper bronze, 4 ( $4^{\prime}$ methoxyphenoxy) toluene, $\mathrm{CH}_{3} \mathrm{O} \cdot \mathrm{C}_{6} \mathrm{H}_{4} \cdot \mathrm{O} \cdot \mathrm{C}_{6} \mathrm{H}_{4} \cdot \mathrm{CH}_{3}$, was formed. On boiling with hydriodic acid, the compound $\mathrm{C}_{13} \mathrm{H}_{12} \mathrm{O}_{2}$ was produced, whilst on boiling with permanganate the toluene compound was oxidised to the corresponding benzoic acid, $\mathrm{C}_{14} \mathrm{H}_{12} \mathrm{O}_{4}$.

The complete synthesis of desiodo-thyroxin was carried out by a slightly different route; parabromanisol was condensed with potassium phenate to give ( $4^{\prime}$ methoxyphenoxy)-benzene, from which, by the hydrocyanic acid method, the aldehyde $\mathrm{C}_{14} \mathrm{H}_{12} \mathrm{O}_{3}$ was obtained. The latter could be oxidised to the acid $\mathrm{C}_{14} \mathrm{H}_{12} \mathrm{O}_{4}$. From the aldehyde both the acid $\mathrm{C}_{16} \mathrm{H}_{14} \mathrm{O}_{4}$ and desiodo-thyroxin were synthesised; two methods were used in the case of the latter. The aldehyde was condensed with glycine anhydride in the presence of acetic anhydride and sodium acetate; the product on boiling with hydriodic acid and red phosphorus underwent simultaneous reduction, demethylation and hydrolysis, with the production of desiodo-thyroxin. In the alternative method the aldehyde was condensed with hydantoin, which on boiling with the same reagents yielded the desired product.

As regards the position of the iodine atoms in thyroxine, the author considers that it is probable that two are present in each benzene ring, in each case lying on either side of and next to the oxygroups, i.e. in the $3,5,3^{\prime}, 5^{\prime}$ positions. The details of the final synthesis will be awaited with interest.

\section{Annual Visitation of the National Physical Laboratory, Teddington.}

$\mathrm{O}^{\mathrm{N}}$ Tuesday, June 22, the General Board of the National Physical Laboratory made its annual visitation of the Laboratory. As in previous years, a large number of members of scientific institutions, technical societies, Government departments, and industrial organisations were also invited to be present. The visitors were received by Sir Ernest Rutherford, president of the Royal Society and chairman of the General Board, and the Director.

The extensive programme of exhibits illustrated well the comprehensive nature of the work which the Laboratory undertakes.

In the I4-foot wind channel a model of the Cierva autogyro, which has aroused so much interest in aeronautical circles, was undergoing test. The model is mounted on three supports forming an inverted pyramid. The vertical forces on the feet of these supports can be measured, enabling the three component forces on the model to be determined, and quantitative data to be obtained for comparison with the conventional type of aeroplane. Another exhibit showed experimental arrangements for the investigation of the spinning characteristics of a model aeroplane. The model under test is mounted on an axis parallel to the wind direction in such a manner that its incidence can be varied. Examination of its behaviour permits the main features of the spinning motion to be studied so that it is possible to indicate what features in design are undesirable from this point of view.

In one of the smaller wind channels experiments were in progress to determine the convective dissipation of heat from the surface of an aerofoil in a wind current. Thin strips of platinum foil cemented to the entire aerofoil and parallel to the span can be heated electrically. Their temperatures can be determined by resistance measurements and equalised by adjustment of the currents traversing them. Measurements of their resistance and of the currents determine the rates of loss of energy from the individual strips.
A new I-foot wind channel has been added to the equipment of the Department. This is intended primarily for the development and calibration of instruments for measuring wind speed and direction.

Among the exhibits of the Metallurgy Department were eight samples of British Standardised Steel, issued jointly by the Iron and Steel Institute and the National Physical Laboratory. These comprised four carbon standards containing respectively 0.10 per cent., 0.27 per cent., 0.65 per cent., and I.09 per cent. of carbon, two sulphur standards containing respectively 0.027 per cent. and $0.07 \mathrm{I}$ per cent. of sulphur, one standard containing phosphorus ( $\mathrm{O} \cdot 029$ per cent.) and one manganese (0.69 per cent.). These standards are prepared from fine millings taken from six-inch bars. Millings taken from the outer layers and the core are rejected, samples of the remainder being subjected to analysis by the Laboratory and various co-operating authorities.

Specimens of pure elements prepared in the Department were also on view. Mention may be made of two of these with which some advance has been made, namely, beryllium and silicon. The former is prepared by the electrolysis of a mixture of the fluorides of beryllium, sodium, and barium, a rotating cathode being used. This is slowly withdrawn at a predetermined rate with the pure metal adhering. Special precautions are taken to prevent contamination of the metal by enclosing the salts in special crucibles. Specimens of these made of magnesia and alumina were shown in the foundry. For work on the constitution of silicon-aluminium alloys and on age hardening in aluminium alloys, very pure silicon is required. Samples were shown of 99.8 per cent. purity. Another exhibit illustrated the microscopic examination of metals usually liquid at ordinary temperatures. Such materials as are used in dental practice are included in this category. By surrounding them with a freezing mixture of carbon dioxide snow and acetone, it is possible to polish the surface to the degree requisite for microscopic examination. 